\title{
Impact of Chronic Kidney Disease on Outcomes in Transcatheter Aortic Valve Implantation
}

Jonathan JL Yap, $,{ }^{* 1} M B B S, M R C P, M P H$, Julian CK Tay, ${ }^{*}{ }^{1} M B B S$, MRCP, MMed (Int Med), See Hooi Ewe, ${ }^{1} M B B S, M R C P, P h D$, Nishanth Thiagarajan, ${ }^{1} M B B S$, Shaw Yang Chia, ${ }_{B S S C}$, Mohammed Rizwan Amanullah, ${ }^{1}$ MBBS, MRCP, FAMS, Soo Teik Lim, ${ }^{1}$ MBBS, MRCP, FAMS, Victor TT Chao, ${ }^{2}$ MBBS, FRCS, FAMS, Kay Woon $\underline{\mathrm{Ho}},{ }^{1}$ MBBS, MRCP, FAMS

\begin{abstract}
Introduction: Chronic kidney disease (CKD) is a significant comorbidity in aortic stenosis (AS) patients. We examined the impact of baseline CKD, postoperative acute kidney injury (AKI) and CKD progression on clinical outcomes in patients who underwent transcatheter aortic valve implantation (TAVI). Materials and Methods: Consecutive patients with severe AS who underwent TAVI were classified into CKD stages $1-2\left(\geq 60 \mathrm{~mL} / \mathrm{min} / 1.72 \mathrm{~m}^{2}\right), 3\left(30-59 \mathrm{~mL} / \mathrm{min} / 1.73 \mathrm{~m}^{2}\right)$ and $4-5\left(<30 \mathrm{~mL} / \mathrm{min} / 1.73 \mathrm{~m}^{2}\right.$ or dialysis) based on estimated glomerular filtration rate (eGFR). Primary outcome was mortality and secondary outcomes included 1-year echocardiographic data on aortic valve area (AVA), mean pressure gradient (MPG) and aortic regurgitation (AR). Results: A total of 216 patients were included. Higher eGFR was associated with lower overall mortality (adjusted

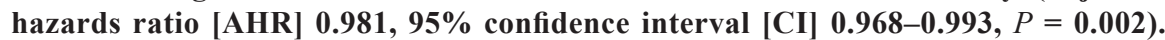
CKD 4-5 were associated with significantly higher mortality from noncardiovascular causes $(P<0.05)$. Patients with CKD 3-5 had higher incidence of moderate AR than those with CKD 1-2 $(P=\mathbf{0 . 0 1 0})$; no difference in AVA and MPG was seen. AKI patients had higher mortality $(P=\mathbf{0 . 0 0 8})$, but the effect was attenuated on multivariate analysis (AHR 1.823, 95\% CI $0.977-3.403, P=\mathbf{0 . 0 5 9}$ ). Patients with CKD progression also had significantly higher mortality (AHR 2.969, 95\% CI 1.373-6.420, $P=0.006)$. Conclusion: CKD in severe AS patients undergoing TAVI portends significantly higher mortality and morbidity. Renal disease progression impacts negatively on outcomes and identifies a challenging subgroup of patients for optimal management.
\end{abstract}

Ann Acad Med Singapore 2020;49:273-84

Key words: Acute kidney injury, Aortic stenosis, Transcatheter aortic valve replacement

\section{Introduction}

With improvements in device technology as well as increasing experience, transcatheter aortic valve implantation (TAVI) has replaced open surgical aortic valve implantation as the treatment of choice in severe symptomatic aortic stenosis (AS) patients who have prohibitive and high surgical risks. ${ }^{1,2}$ TAVI has also gained increasing uptake in patients with intermediate surgical risk. ${ }^{3}$

Chronic kidney disease (CKD) is an established disease modifier in most major cardiovascular diseases ${ }^{4}$ and portends significant mortality and morbidity in patients undergoing TAVI. Patients who have prohibitive and high surgical risks are known to have multiple comorbidities, of which CKD is prevalent. ${ }^{4}$ However, in many landmark TAVI trials such as PARTNER, PARTNER 2 or CoreValve, only a minority (about $5 \%$ ) of patients have baseline serum creatinine $>2 \mathrm{mg} / \mathrm{dL}$ and end-stage renal failure (ESRF) patients were generally excluded. ${ }^{1,2,5,6}$ Additionally, little information is available on the effects of postoperative acute kidney injury (AKI) and long-term renal function

\footnotetext{
${ }^{1}$ Department of Cardiology, National Heart Centre Singapore, Singapore

${ }^{2}$ Department of Cardiothoracic Surgery, National Heart Centre Singapore, Singapore

*Both authors contributed equally to the initial concept/design, acquisition, analysis and interpretation of data, drafting of manuscript and validation of final manuscript.

Address for Correspondence: Dr Ho Kay Woon, Department of Cardiology, National Heart Centre Singapore, 5 Hospital Drive, Singapore 169609.

Email: ho.kay.woon@singhealth.com.sg
} 
trajectory on valve haemodynamics and clinical outcomes. This study aimed to evaluate the impact of baseline CKD status, postoperative AKI and CKD progression on early and late outcomes and valve haemodynamics in severe AS patients undergoing TAVI.

\section{Materials and Methods}

This is a prospective registry of all consecutive patients with severe symptomatic AS who underwent TAVI in a single tertiary cardiac centre from October 2009 to August 2017. A heart team that comprised cardiothoracic surgeons, interventional cardiologists and cardiac imaging physicians were involved in the selection of patients, transcatheter valve type and study approach. Registry participation did not impact on clinical management. Written informed consent was obtained from all patients and the study was approved by the Institutional Review Board.

TAVI was performed according to previously published standard protocol. ${ }^{6,7}$ After discharge, clinical review was done at 30 days, 3 months, 12 months and annually thereafter. All patients underwent echocardiographic evaluation at baseline prior to intervention and discharge, at follow-up 3 months later and yearly thereafter. Serum creatinine and estimated glomerular filtration rate (eGFR) were evaluated preoperatively, at $24-48$ hours postoperatively and at similar intervals after discharge.

Using Cockcroft-Gault formula, eGFR was calculated based on serum creatinine. CKD was classified into 5 stages according to the guidelines of the Kidney Disease: Improving Global Outcomes workgroup ${ }^{8}$ who used eGFR to determine them. The 5 stages are CKD 1 $\left(\right.$ eGFR $\left.\geq 90 \mathrm{~mL} / \mathrm{min} / 1.72 \mathrm{~m}^{2}\right)$, CKD 2 (eGFR $69-89 \mathrm{~mL} /$ $\left.\mathrm{min} / 1.72 \mathrm{~m}^{2}\right)$, CKD $3\left(\mathrm{eGFR} \geq 30-59 \mathrm{~mL} / \mathrm{min} / 1.72 \mathrm{~m}^{2}\right)$, CKD 4 (eGFR $\left.\geq 15-29 \mathrm{~mL} / \mathrm{min} / 1.72 \mathrm{~m}^{2}\right)$ and CKD 5 or ESRF $\left(e G F R<15 \mathrm{~mL} / \mathrm{min} / 1.72 \mathrm{~m}^{2}\right) .{ }^{8}$ Advanced CKD is defined as CKD 4 and above.

AKI was defined according to the Valve Academic Research Consortium (VARC) consensus on event definition (modified Risk, Injury, Failure, Loss of kidney function and End-stage kidney disease classification) as an absolute increase in serum creatinine of $>0.3 \mathrm{mg} / \mathrm{dL}$ or an increase of $>50 \%$ within 72 hours following TAVI. ${ }^{9}$ Patients who developed AKI were classified according to severity into stage 1 (creatine $150-200 \%$ or $>0.3 \mathrm{mg} / \mathrm{dL}$ ), stage 2 (creatinine $200-300 \%$ ) or stage 3 (creatinine $>300 \%$, creatinine $>4.0 \mathrm{mg} / \mathrm{dL}$ with an increase of at least $0.5 \mathrm{mg} / \mathrm{dL}$ or require renal replacement therapy).
At 3 months, repeat renal panel was performed. Renal disease progression was defined as an increase in CKD stage from baseline or new requirement for renal replacement therapy.

During hospitalisation, operative success and major perioperative complications from TAVI were assessed. Echocardiographic outcomes were analysed at discharge and 12 months and included aortic valve (AV) area, mean AV pressure gradient and AV regurgitation (graded as none/trace, mild, moderate and severe). Mortality and its aetiology (cardiovascular vs non-cardiovascular) were obtained from national registries and classified into early (up to 30 days) and cumulative (inclusive of 30 days until last follow-up) mortality. All outcomes were defined according to VARC-2 criteria. ${ }^{10}$

Continuous variables were subjected to 1-way analysis of variance and results were expressed as mean and standard deviation (SD). Categorical variables were analysed using chi-square test and the findings were expressed as counts and percentages. Logistic regression was used to compare outcomes between groups for in-hospital/30-day outcomes; Cox proportional hazards regression was used to analyse cumulative outcomes. Multivariate analysis was used to derive odds ratio (OR) for logistic regression, hazards ratio (HR) for Cox regression and 95\% confidence intervals (CI) for predictive variables. Survival curves were presented. All statistical analyses were performed using SPSS Statistics for Windows, Version 25.0 (IBM Corp., Armonk, NY, USA). A value of $P<0.05$ was considered statistically significant.

\section{Results}

A total of 216 severe symptomatic AS patients who underwent TAVI were included; 55 (25.5\%) were CKD $1-2,100(46.3 \%)$ were CKD 3 and 61 (28.2\%) were CKD 4-5 (24 were on dialysis). Mean and median follow-up were 2.63 years (SD 2.11) and 2.23 years (interquartile range 0.83-4.14 years) years, respectively. Baseline and procedural characteristics according to CKD severity are shown in Tables 1 and 2, respectively.

Patients with advanced CKD were older $(P=0.001)$, had lower body mass index $(P=0.001)$ and poor effort tolerance of at least New York Heart Association (NYHA) class III-IV $(P=0.002)$. No significant differences were noted in baseline cardiovascular risk factors, atrial fibrillation, ischaemic heart disease, prior cerebrovascular accidents or peripheral vascular disease (PVD). There was a commensurate increase in 
Table 1. Baseline Characteristics According to CKD Severity

\begin{tabular}{|c|c|c|c|c|c|}
\hline Variable & $\begin{array}{l}\text { Aggregate } \\
(n=216)\end{array}$ & $\begin{array}{c}\text { CKD } 1-2 \\
(n=55)\end{array}$ & $\begin{array}{c}\text { CKD 3 } \\
(n=100)\end{array}$ & $\begin{array}{l}\text { Advanced CKD } \\
(n=61)\end{array}$ & $P$ Value \\
\hline Mean age, years (SD) & $75.5(9.3)$ & $70.3(8.5)$ & $78.2(7.3)$ & $75.6(11.0)$ & $<0.001$ \\
\hline Male gender $(\%)$ & $106(49.1)$ & $25(45.5)$ & $53(53)$ & $28(45.9)$ & 0.992 \\
\hline Mean body mass index, $\mathrm{kg} / \mathrm{m}^{2}(\mathrm{SD})$ & $23.9(4.7)$ & $26.2(4.6)$ & $23.4(4.2)$ & $22.8(4.9)$ & $<0.001$ \\
\hline $\mathrm{I}-\mathrm{II}$ & $90(41.7)$ & $31(56.4)$ & $42(42)$ & $17(27.9)$ & \\
\hline III - IV & $126(58.3)$ & $24(43.6)$ & $58(58)$ & $44(72.1)$ & \\
\hline Smoker $(\%)$ & $41(19)$ & $8(14.5)$ & $25(25)$ & $8(13.1)$ & 0.788 \\
\hline Diabetes mellitus (\%) & $86(39.8)$ & $25(45.5)$ & $35(35)$ & $26(42.6)$ & 0.793 \\
\hline Hyperlipidaemia (\%) & $165(76.4)$ & $44(80)$ & $72(72)$ & $49(80.3)$ & 0.928 \\
\hline Prior ischaemic heart disease $(\%)$ & $127(58.8)$ & $33(60)$ & $60(60)$ & $34(55.7)$ & 0.634 \\
\hline Prior coronary artery bypass (\%) & $49(22.7)$ & $13(23.6)$ & $20(20)$ & $6(26.2)$ & 0.717 \\
\hline Atrial fibrillation (\%) & $45(20.8)$ & $7(12.7)$ & $24(24)$ & $14(23)$ & 0.189 \\
\hline Prior stroke $(\%)$ & $27(12.5)$ & $4(7.3)$ & $15(15)$ & $8(13.1)$ & 0.362 \\
\hline Peripheral vascular disease (\%) & $35(16.2)$ & $5(9.1)$ & $17(17)$ & $13(21.3)$ & 0.077 \\
\hline Chronic obstructive lung disease (\%) & $22(10.2)$ & $4(7.3)$ & $16(16)$ & $2(3.3)$ & 0.424 \\
\hline Mean eGFR, $\mathrm{mL} / \mathrm{min} / 1.72 \mathrm{~m}^{2}(\mathrm{SD})$ & $45.8(26.1)$ & $80.2(19.5)$ & $44.3(8.1)$ & $17.3(8.4)$ & $<0.001$ \\
\hline Mean STS risk score (SD) & $6.5(6.1)$ & $3.5(2.1)$ & $6.1(7)$ & $9.8(5.2)$ & $<0.001$ \\
\hline Mean EuroSCORE II (SD) & $6.2(7.6)$ & $3.4(3.1)$ & $5.8(8.0)$ & $9.4(8.5)$ & $<0.001$ \\
\hline Mean AV calcium score in Agatston units, $n=104(S D)$ & $2634(1943)$ & $2153(1041)$ & $2690(1820)$ & $3045(2804)$ & 0.046 \\
\hline
\end{tabular}

AV: Aortic valve; CKD: Chronic kidney disease; eGFR: Estimated glomerular filtration rate; EuroSCORE: European System for Cardiac Operative Risk Evaluation; NYHA: New York Heart Association; SD: Standard deviation; STS: Society of Thoracic Surgeons

surgical risk on the Society of Thoracic Surgeons risk score, Logistic European System for Cardiac Operative Risk Evaluation (EuroSCORE) and EuroSCORE II with increasing CKD severity $(P=0.001)$. CKD severity was also associated with heavier AV calcification on AV calcium score $(P=0.046)$. For procedural characteristics, no significant differences were noted in the different stages of CKD severity in TAVI approach, size and type of prostheses and procedural contrast volume used.

Findings of univariate analysis showed that CKD 1-2 (OR $0.192,95 \%$ CI $0.040-0.921, P=0.039$ ) and CKD 3 (OR $0.052,95 \%$ CI $0.006-0.414, P=0.005$ ) were associated with significantly lower 30 -day mortality than advanced CKD. After adjusting for diabetes mellitus, PVD and non-transfemoral TAVI approach, findings of multivariate analysis showed that the relationship was attenuated in CKD 1-2 patients against advanced CKD patients (adjusted OR [AOR] $0.257,95 \%$ CI $0.047-1.390, P=0.115$ ); however, it remained significant in CKD 3 patients vs advanced CKD patients (AOR 0.047, 95\% CI $0.005-0.414, P=0.006$ ). Higher eGFR was also significantly associated with lower 30-day mortality (OR $0.967,95 \%$ CI $0.939-0.996, P=0.024$ ), but the effect was similarly attenuated on multivariate analysis (AOR $0.969,95 \%$ CI $0.938-1.000, P=0.053$ ). At 30 days, CKD 3 was associated with significantly 
Table 2. Procedural Characteristics According to CKD Severity

\begin{tabular}{|c|c|c|c|c|}
\hline Variable & $\begin{array}{c}\text { CKD } 1-2 \\
(n=55)\end{array}$ & $\begin{array}{c}\text { CKD } 3 \\
(n=100)\end{array}$ & $\begin{array}{l}\text { Advanced CKD } \\
\qquad(n=61)\end{array}$ & $P$ Value \\
\hline Approach (\%) & & & & 0.414 \\
\hline Transfemoral & $44(80)$ & $79(79)$ & $45(73.8)$ & \\
\hline Non-transfemoral & $11(20)$ & $21(21)$ & $16(26.2)$ & \\
\hline Trans-apical & $7(12.7)$ & $18(18)$ & $11(18)$ & \\
\hline Direct-aortic & $3(5.5)$ & $3(3)$ & $5(8.2)$ & \\
\hline Trans-subclavian & $1(1.8)$ & $0(0)$ & $0(0)$ & \\
\hline Prosthesis type (\%) & & & & 0.959 \\
\hline Self-expandable & $24(43.6)$ & $47(47)$ & $27(44.3)$ & \\
\hline Valve generation $(\%)^{*}$ & & & & 0.613 \\
\hline Early & $45(93.8)$ & $86(90.5)$ & $52(88.1)$ & \\
\hline New & $3(6.3)$ & $9(9.5)$ & $7(11.9)$ & \\
\hline Prosthesis size (\%) & & & & 0.247 \\
\hline $23 \mathrm{~mm}$ & $19(35.2)$ & $34(34)$ & $26(42.5)$ & \\
\hline $25 \mathrm{~mm}$ & $2(3.7)$ & $2(2)$ & $1(1.6)$ & \\
\hline $26 \mathrm{~mm}$ & $18(33.3)$ & $47(47)$ & $23(37.7)$ & \\
\hline $27 \mathrm{~mm}$ & $1(1.9)$ & $0(0)$ & $0(0)$ & \\
\hline $29 \mathrm{~mm}$ & $11(20.4)$ & $16(16)$ & $9(14.8)$ & \\
\hline Mean contrast volume, mL (SD) & $139(68)$ & $143(77)$ & $116(56)$ & 0.070 \\
\hline Device success $(\%)$ & $53(96.4)$ & $95(95)$ & $60(98.4)$ & 0.549 \\
\hline
\end{tabular}

CKD: Chronic kidney disease; SD: Standard deviation

"Early-generation valves refer to CoreValve, SAPIEN and SAPIEN XT. New-generation valves refer to CoreValve Evolut R, CoreValve Evolut Pro and SAPIEN 3. A total of 8 Lotus, 3 Portico and 2 Engager valve cases were excluded from analysis.

lower odds of new permanent pacemaker implantation (PPM) than advanced CKD (3\% vs $13.1 \%$, OR 0.205 , $95 \%$ CI $0.052-0.805, P=0.023)$. A trend towards lower odds in new PPM implantation in CKD 1-2 vs advanced CKD (3.6\% vs $13.1 \%$, OR $0.250,95 \%$ CI $0.051-1.233, P=0.089)$ patients was observed. No significant differences were seen in length of hospitalisation, major vascular complications, stroke or bleeding rates (Table 3 ).

At 1 year, the mortality rates in CKD $1-2$, CKD 3 and advanced CKD patients were $9.1 \%, 9 \%$ and $23 \%$, respectively; at 3 years, the overall mortality rates in the 3 groups were $16.4 \%, 24 \%$ and $45.9 \%$, respectively.
In patients who were on dialysis, the 1- and 3-year mortality rates were $16.7 \%$ and $50 \%$, respectively. After adjusting for left ventricular ejection fraction (LVEF) and NYHA status, findings of multivariate analysis showed that CKD 1-2 (adjusted HR [AHR] 0.366, 95\% CI 0.168-0.797, $P=0.011$ ) and CKD 3 (AHR 0.467, $95 \%$ CI $0.267-0.817, P=0.008$ ) were significantly associated with lower overall mortality than advanced CKD. Higher eGFR was also associated with lower overall mortality (AHR 0.981, 95\% CI 0.968-0.993, $P=0.002$ ). Increased mortality (Fig. 1) was attributed to non-cardiovascular causes in CKD 1-2 vs advanced CKD (AHR 0.360, 95\% CI 0.132-0.979, $P=0.045$ ) 
Table 3. Outcome at 30 Days According to CKD Severity

\begin{tabular}{|c|c|c|c|c|}
\hline Variable & $\begin{array}{c}\text { CKD 1 } 1-2 \\
(n=55)\end{array}$ & $\begin{array}{c}\text { CKD 3 } \\
(n=100)\end{array}$ & $\begin{array}{l}\text { Advanced CKD } \\
\quad(n=61)\end{array}$ & $P$ Value \\
\hline Mean hospital stay in days (SD) & $9.8(14.5)$ & $9.9(10.7)$ & $11.4(11.7)$ & 0.711 \\
\hline 30-day mortality (\%) & $2(3.6)$ & $1(1)$ & $10(16.4)$ & 0.003 \\
\hline Major vascular complications (\%) & $6(10.9)$ & $11(11)$ & $11(18)$ & 0.244 \\
\hline Major bleeding (\%) & $4(7.3)$ & $4(4)$ & $5(8.2)$ & 0.803 \\
\hline Minor bleeding (\%) & $2(3.6)$ & $10(10)$ & $6(9.8)$ & 0.240 \\
\hline Stroke (\%) & $1(1.8)$ & $1(1)$ & $0(0)$ & 0.307 \\
\hline New pacemaker $(\%)$ & $2(3.6)$ & $3(3)$ & $8(13.1)$ & 0.038 \\
\hline Acute kidney injury (\%) & & & & 0.008 \\
\hline Total & $3(5.5)$ & $19(19)$ & $15(40.5)^{*}$ & \\
\hline Stage 1 & $2(3.6)$ & $10(10)$ & $9(24.3)^{*}$ & \\
\hline Stage 2 & $1(1.9)$ & $1(1)$ & $2(5.4)^{*}$ & \\
\hline Stage 3 & $0(0)$ & $8(8)$ & $4(10.8)^{*}$ & \\
\hline Dialysis & $0(0)$ & $5(5)$ & $4(10.8)^{*}$ & \\
\hline
\end{tabular}

CKD: Chronic kidney disease; SD: Standard deviation

"Exclude 24 patients who were already on dialysis.

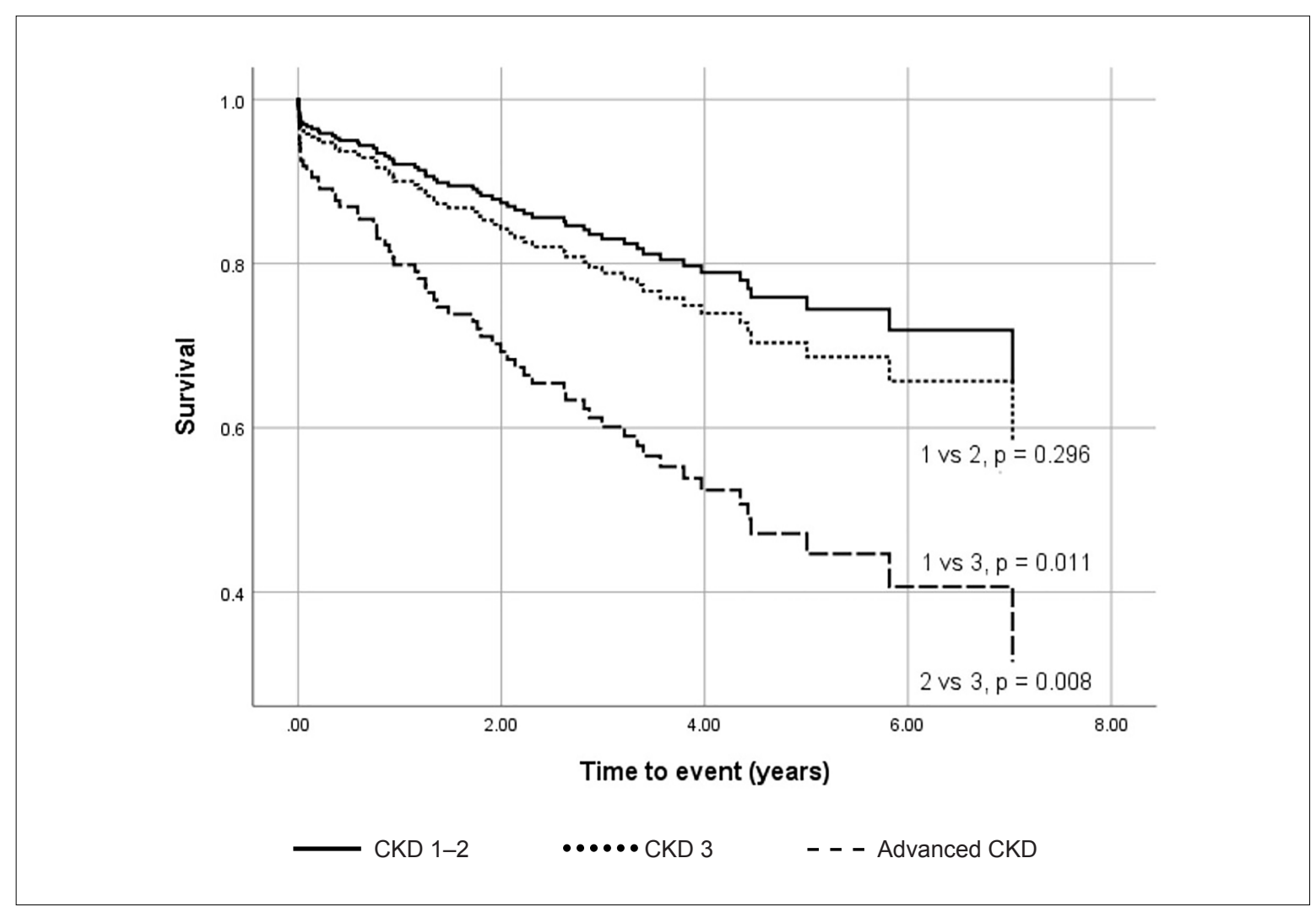

Fig. 1. Survival curves for cumulative overall mortality in patients with chronic kidney disease (CKD) stages 1-2 (solid line), stage 3 (dotted line) and advanced stages (4-5) or end-stage renal failure (dashed line). 
patients and in CKD 3 vs advanced CKD (AHR 0.314, $95 \%$ CI $0.146-0.675, P=0.003$ ) patients, particularly with progression of kidney disease $(P=0.008)$ and non-respiratory sepsis $(P=0.003)$ (Table 4$)$.

At 1-year post-TAVI, echocardiographic outcomes were available in $173(80.1 \%)$ patients; no significant differences in valve area and mean transvalvular valve gradient were observed across all CKD groups. However, CKD 3 (20\%) and CKD 4-5 (22\%) patients had higher incidence of AR of at least moderate severity than CKD 1-2 $(2.2 \%)$ patients $(P=0.010)$. Except for 1 case of transvalvular regurgitation, all AR cases had paravalvular regurgitation (Table 4).

Seven patients experienced severe TAVI valve leaflet degeneration; 6 were restenosis and 1 was mixed stenosis with regurgitation. Two patients were on dialysis and the remaining 5 were in CKD 1-3. In the dialysis patients, valve degeneration occurred at between 1.5-2 years; in remaining patients, it occurred at between 3.75-6 years. Three patients underwent repeat TAVI and another 3 passed away. Six patients underwent either a transoesophageal echocardiogram and/or computed tomography, except for 1 patient who did not undergo further investigations due to poor premorbid status. In 5 of them, thrombosis was ruled out and they were not anticoagulated. In 1 patient, embolic phenomenon was suspected and low molecular weight heparin was initiated, but patient passed away during that admission (Table 5).

After excluding patients who were already on dialysis, 37 (19.3\%) patients developed AKI postTAVI; $21(10.9 \%), 4(2.1 \%)$ and $12(6.3 \%)$ patients were in AKI stages 1, 2 and 3, respectively. Among AKI stage 3 patients, 4 required dialysis. No significant differences were noted in amount of contrast volume used during TAVI in AKI (mean $133 \mathrm{~mL}, \mathrm{SD}$ 64) and non-AKI (mean $140 \mathrm{~mL}$, SD 96) patients $(P=0.113)$. A significant association between severity of baseline CKD (OR 5.014, 95 CI 1.074-23.403, $P=0.040)$ and occurrence of AKI was seen. Findings of univariate analysis showed that AKI patients had higher overall mortality $(43.2 \%$ vs $25.1 \%$, HR 2.275 , $95 \%$ CI $1.237-4.185, P=0.008$ ), but the effect was attenuated after adjustment for LVEF and NYHA status (AHR 1.823, 95\% CI 0.977-3.403, $P=0.059$ ). No significant interaction was found for mortality $\left(\mathrm{p}_{\text {interaction }}=0.851\right)$ between AKI and baseline CKD status (Fig. 2).

After patients who were already on dialysis were excluded, findings of renal panel at 3 months showed that $138(71.9 \%)$ patients had stable CKD and $37(19.3 \%)$ patients had progressive CKD. In patients

Table 4. Cumulative Outcomes According to CKD severity

\begin{tabular}{|c|c|c|c|c|c|c|c|}
\hline \multirow[t]{2}{*}{ Variable } & \multicolumn{2}{|c|}{ CKD $1-2$} & \multicolumn{2}{|c|}{ CKD 3} & \multicolumn{2}{|c|}{ Advanced CKD } & \multirow[t]{2}{*}{$P$ Value } \\
\hline & $N=46$ & $\mathbf{N}=\mathbf{5 5}$ & $\mathbf{N}=\mathbf{8 2}$ & $\mathbf{N}=\mathbf{1 0 0}$ & $N=45$ & $N=61$ & \\
\hline \multicolumn{8}{|l|}{ 1-year echocardiographic outcomes } \\
\hline Mean AV area (SD) & $1.57(0.38)$ & & $1.58(0.38)$ & & $1.69(0.49)$ & & 0.290 \\
\hline Mean AV pressure gradient, $\mathrm{mmHg}$ (SD) & $12.6(5.9)$ & & $11.6(5.8)$ & & $11.2(6.0)$ & & 0.494 \\
\hline$\geq 2+$ aortic regurgitation $(\%)$ & $1(2.2)$ & & $18(22)$ & & $9(20)$ & & 0.021 \\
\hline Overall mortality & & $9(16.4)$ & & $24(24)$ & & $28(45.9)$ & $<0.001$ \\
\hline Cardiovascular mortality & & $4(7.3)$ & & $14(14)$ & & $8(13.1)$ & 0.447 \\
\hline Non-cardiovascular mortality & & $5(9.1)$ & & $10(10)$ & & $20(32.8)$ & 0.006 \\
\hline Respiratory & & $0(0)$ & & $3(3)$ & & $2(3.3)$ & 0.414 \\
\hline Kidney failure & & $1(1.8)$ & & $0(0)$ & & $5(8.2)$ & 0.008 \\
\hline Bleeding & & $0(0)$ & & $1(0)$ & & $0(0)$ & 0.558 \\
\hline Non-respiratory sepsis & & $1(1.8)$ & & $2(2)$ & & $8(13.1)$ & 0.003 \\
\hline
\end{tabular}

AV: Aortic valve; CKD: Chronic kidney disease; SD: Standard deviation 


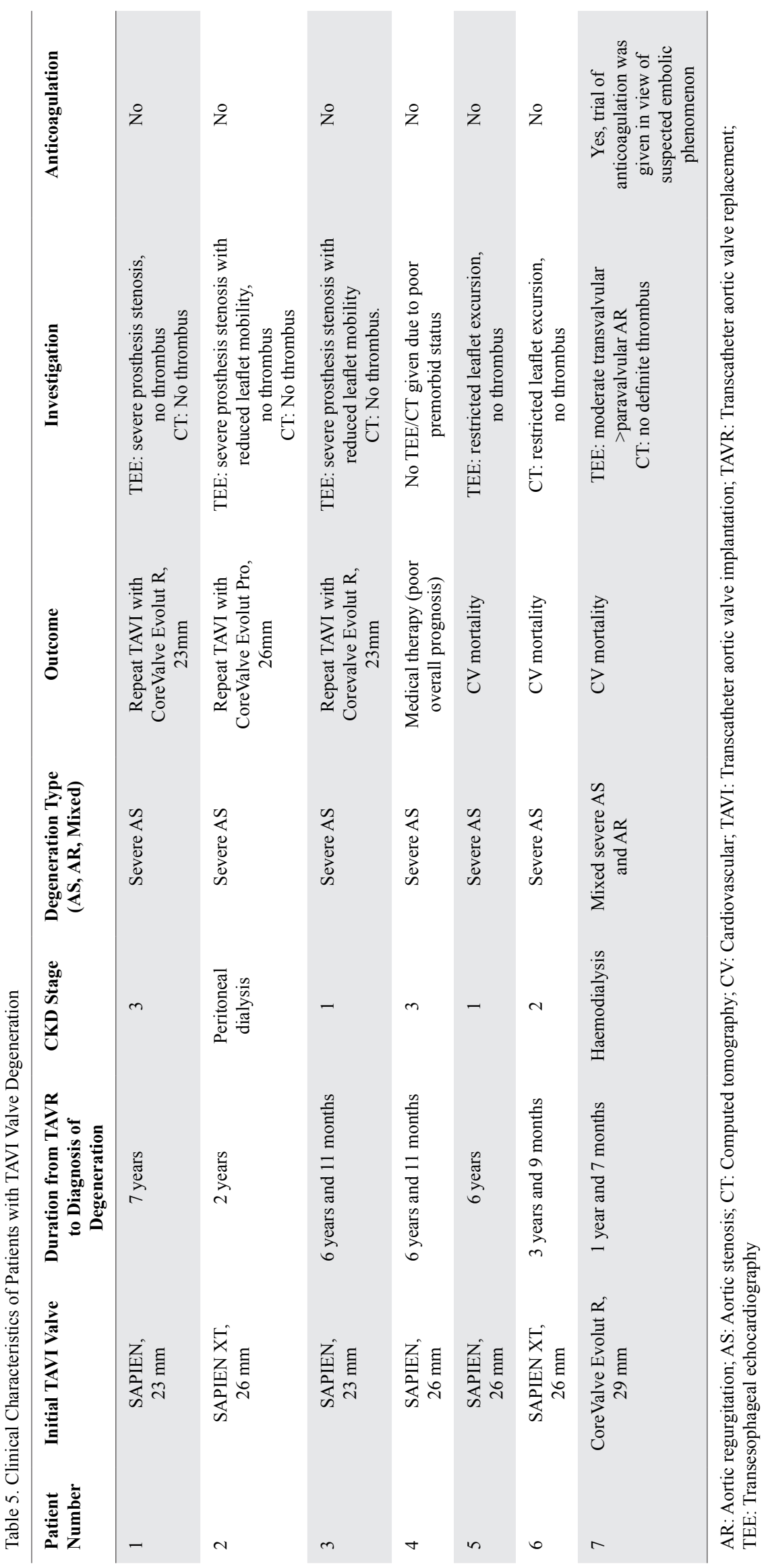




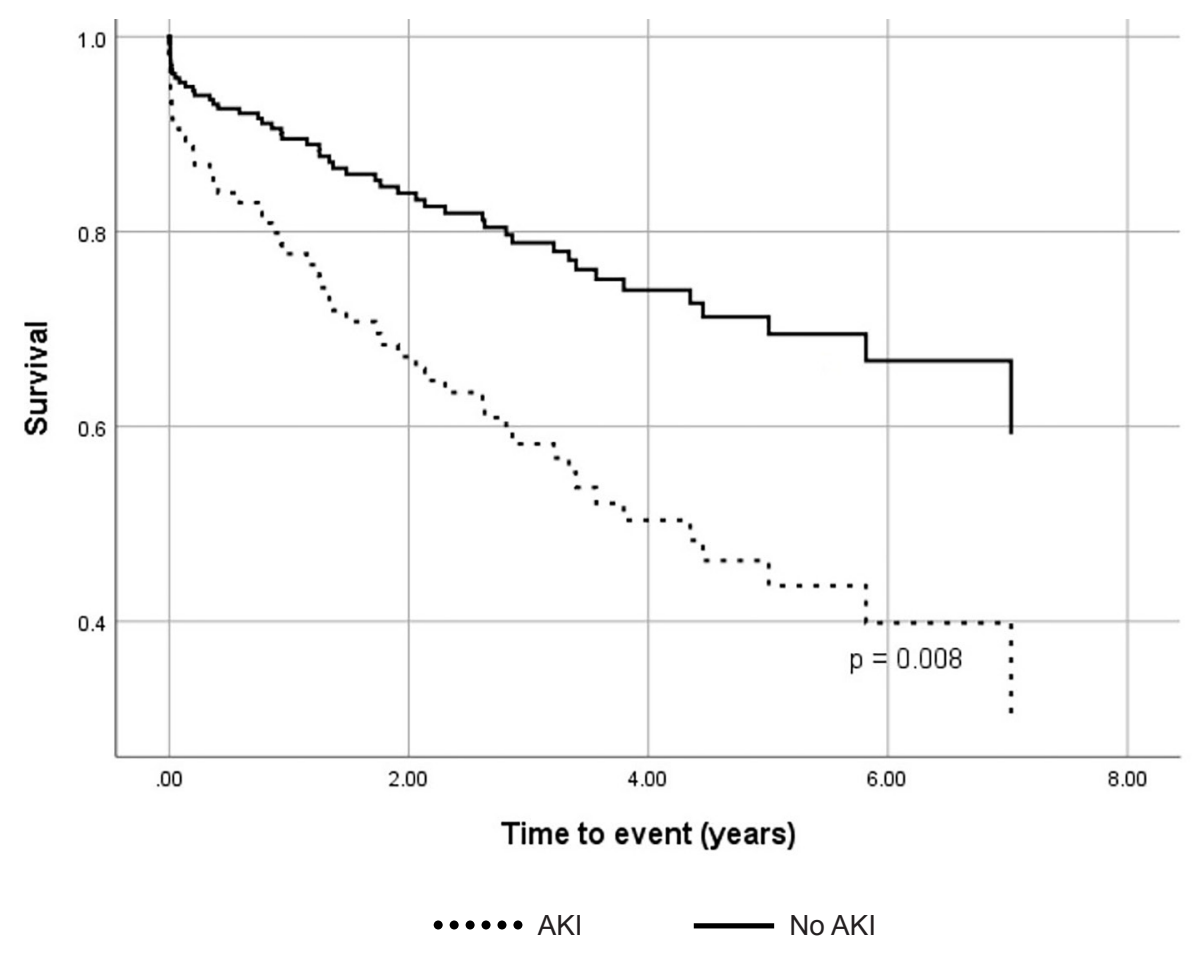

Fig. 2. Survival curves for cumulative overall mortality between acute kidney injury (AKI) patients (dotted line) and non-AKI patients (solid line).

with progressive CKD, findings of multivariate analysis revealed that they had significantly higher overall mortality (AHR 2.883, 95\% CI 1.321$6.290, P=0.008)$. No significant interaction was found for mortality $\left(\mathrm{p}_{\text {interaction }}=0.157\right)$ between CKD progression and baseline CKD status.

In this study, $19(8.8 \%)$ patients underwent TAVI with new-generation valves that included CoreValve Evolut R (Medtronic Inc., Minneapolis, MN, USA), CoreValve Evolut PRO (Medtronic Inc., Minneapolis, MN, USA) and SAPIEN 3 (Edwards Lifesciences Corp., Irvine, CA, USA). No significant differences in the use of new-generation valves were found among CKD 1-2 (6.3\%), CKD 3 (9.5\%) and CKD 4-5/ESRF $(11.9 \%)$ patients $(P=0.613)$. Additionally, newer-generation valves did not impact any early or late outcomes (Table 6).

No significant differences in the use of self-expandable valves (SEV) and balloon-expandable valves (BEV) were seen in CKD patients $(P=0.959)$. Mean contrast volume was $123 \mathrm{~mL}$ (SD 76) in BEV, which was significantly lower than $146 \mathrm{~mL}$ (SD 62) in SEV
$(P=0.020)$. At 1 year, echocardiographic studies showed that AV area was lower in BEV (mean 1.50, SD 0.35) than SEV (mean 1.72, SD 0.44, $P=0.001$ ); $\mathrm{AV}$ gradient was also higher in BEV (mean $12.9 \mathrm{mmHg}$, SD 5.1) than SEV (mean $10.4 \mathrm{mmHg}$, SD 6.5, $P=0.001$ ). No significant differences were observed in development of moderate AR and pacemaker, stroke and mortality rates (Table 7).

\section{Discussion}

This study evaluated the impact of baseline CKD status, postoperative AKI and CKD progression on early and late outcomes and valve haemodynamics in severe AS patients undergoing TAVI. The significant findings included: 1) CKD had a negative impact on cumulative overall mortality that was attributed to non-cardiovascular mortality and this effect was seen as early as at 30 days, but was more pronounced on long-term follow-up; 2) CKD resulted in significantly higher AR and PPM implantation rates; 3) postoperative AKI had a negative impact on overall mortality, but this effect was attenuated after adjustment for 
Table 6. Valve Haemodynamic Outcomes in Early- and New-Generation TAVI Valves at 30 Days and 1 Year

\begin{tabular}{|c|c|c|c|c|c|c|c|}
\hline \multirow[t]{2}{*}{ Variable } & \multicolumn{3}{|c|}{ Early-Generation Valves } & \multicolumn{3}{|c|}{ New-Generation Valves } & \multirow[t]{2}{*}{$P$ Value } \\
\hline & $N=183$ & $N=164$ & $\mathbf{N}=147$ & $\mathbf{N}=19^{*}$ & $\mathbf{N}=15$ & $N=14$ & \\
\hline Mean contrast volume, mL (SD) & $126(65)$ & & & $163(54)$ & & & 0.021 \\
\hline 30-day mortality (\%) & $13(7.1)$ & & & $0(0)$ & & & 0.230 \\
\hline Stroke $(\%)$ & $1(0.5)$ & & & $0(0)$ & & & 0.747 \\
\hline New pacemaker $(\%)$ & $11(6.0)$ & & & $0(0)$ & & & 0.272 \\
\hline Acute kidney injury $(\%)^{\dagger}$ & & & & & & & 0.219 \\
\hline Total & & $32(19.5)$ & & & $1(6.7)$ & & \\
\hline Stage 1 & & $18(11)$ & & & $0(0)$ & & \\
\hline Stage 2 & & $3(1.8)$ & & & $0(0)$ & & \\
\hline Dialysis & & $8(4.9)$ & & & $1(6.7)$ & & \\
\hline 1-year overall mortality & $24(13.1)$ & & & $1(5.3)$ & & & 0.323 \\
\hline 1-year cardiovascular mortality & $11(6.0)$ & & & $0(0)$ & & & 0.272 \\
\hline \multicolumn{8}{|l|}{ 1-year echocardiographic outcomes } \\
\hline Mean AV area (SD) & & & $1.62(0.42)$ & & & $1.69(0.28)$ & 0.527 \\
\hline Mean AV pressure gradient, mmHg (SD) & & & $11.6(5.9)$ & & & $10.2(3.8)$ & 0.381 \\
\hline$\geq 2+$ aortic regurgitation $(\%)$ & & & $22(15.0)$ & & & $2(14.3)$ & 0.946 \\
\hline
\end{tabular}

AV: Aortic valve; SD: Standard deviation; TAVI: Transcatheter aortic valve implantation

*A total of 8 Lotus, 3 Portico and 2 Engager valve cases were excluded from analysis.

${ }^{\dagger}$ Exclude 24 patients who were already on dialysis.

confounders; and 4) renal disease progression was independently associated with higher overall mortality.

CKD can portend worse outcomes in patients who undergo TAVI. ${ }^{5}$ Several studies have established that CKD severity prognosticates acute and long-term mortality. ${ }^{11-15}$ In their study of 41,025 patients who underwent TAVI, Gupta et al reported higher in-hospital mortality in CKD and ESRF patients than non-CKD patients. ${ }^{16}$ In their study of CKD 1-2, CKD 3 and advanced CKD patients, Allende et al found a significant difference in mortality of $15.6 \%, 20 \%$ and $27.5-35.5 \%$, respectively, at 1 year. ${ }^{17}$ Similarly, this study found that advanced CKD was associated with higher mortality at 30 days and more salient differences were observed on long-term follow-up; at 1 year, the mortality rates were $9.1 \%, 9 \%$ and $23 \%$ in CKD $1-2$, CKD3 and advanced CKD patients, respectively.

Dialysis has been shown to be a marker of worse outcomes. ${ }^{18}$ In their study, Allende et al reported slightly higher mortality of $20 \%$ at 1 year and up to approximately $65 \%$ at 3 years in ESRF patients. ${ }^{17}$ In their study of 66 dialysis patients who underwent TAVI, Codner et al also noted higher risk of mortality of close to $24.2 \%$ at 1 year. ${ }^{19}$ In our dialysis patients, the mortality rates at 1 and 3 years were $16.7 \%$ and $50 \%$, respectively.

In patients with advanced CKD, this study found higher non-cardiovascular mortality rates that were attributed to non-respiratory sepsis and renal disease progression, a finding similar to that of Allende et al. ${ }^{17}$ Patients with renal disease are at higher risk of sepsis than the general population ${ }^{19}$ and the reasons include reduced immunity and vaccine efficacy, increased comorbidities, more visits to healthcare facilities and treatment of the disease itself. ${ }^{21}$ CKD is a wellestablished risk factor for cardiovascular disease and mortality. ${ }^{20,22,23}$ Although other studies had found higher cardiovascular mortality rates in TAVI patients with 
Table 7. Valve Haemodynamic Outcomes in BEV and SEV at 30 Days and 1 Year

\begin{tabular}{|c|c|c|c|c|c|c|c|}
\hline \multirow[t]{2}{*}{ Variable } & \multicolumn{3}{|c|}{ SEV } & \multicolumn{3}{|c|}{ BEV } & \multirow[t]{2}{*}{$P$ Value } \\
\hline & $\mathbf{N}=\mathbf{9 8}$ & $N=86$ & $\mathbf{N}=77$ & $N=118$ & $N=106$ & $N=96$ & \\
\hline Mean contrast volume, mL (SD) & $146(62)$ & & & $123(76)$ & & & 0.020 \\
\hline 30-day mortality (\%) & $2(2.0)$ & & & $11(9.3)$ & & & 0.025 \\
\hline Stroke $(\%)$ & $1(1.0)$ & & & $1(0.8)$ & & & 0.895 \\
\hline New pacemaker $(\%)$ & $9(9.2)$ & & & $4(3.4)$ & & & 0.075 \\
\hline Acute kidney injury $(\%)^{*}$ & & & & & & & 0.429 \\
\hline Total & & $14(16.3)$ & & & $22(20.8)$ & & \\
\hline Stage 1 & & $7(8.1)$ & & & $13(12.3)$ & & \\
\hline Stage 2 & & $1(1.2)$ & & & $3(2.8)$ & & \\
\hline Dialysis & & $4(4.7)$ & & & $5(4.7)$ & & \\
\hline 1-year overall mortality & $12(12.2)$ & & & $16(13.6)$ & & & 0.775 \\
\hline 1-year cardiovascular mortality & $6(6.1)$ & & & $6(5.1)$ & & & 0.740 \\
\hline Cumulative overall mortality & $24(24.5)$ & & & $37(31.4)$ & & & 0.264 \\
\hline Cumulative cardiovascular mortality & $13(13.3)$ & & & $13(11.0)$ & & & 0.613 \\
\hline \multicolumn{8}{|l|}{ 1-year echocardiographic outcomes } \\
\hline Mean AV area (SD) & & & $1.72(0.44)$ & & & $1.50(0.35)$ & 0.001 \\
\hline Mean AV pressure gradient, $\mathrm{mmHg}(\mathrm{SD})$ & & & $10.4(6.5)$ & & & $12.9(5.1)$ & 0.001 \\
\hline$\geq 2+$ aortic regurgitation $(\%)$ & & & $14(18.2)$ & & & $14(14.6)$ & 0.523 \\
\hline
\end{tabular}

AV: Aortic valve; BEV: Balloon-expandable valve; SD: Standard deviation; SEV: Self-expandable valve

*Exclude 24 patients who were already on dialysis.

advanced $\mathrm{CKD},{ }^{17,19}$ an insignificant trend was found by this study that could be attributed to smaller sample size.

Advanced CKD has been linked to platelet dysfunction and coagulopathy that contribute to higher risk of bleeding events, especially the use of dual antiplatelet agents or vitamin $\mathrm{K}$ antagonists..$^{24,25}$ Unlike other studies, ${ }^{13,26}$ this study did not show an increase in minor or major bleeding events in patients with advanced CKD at 30 days. A longer duration of followup is needed to evaluate differences in the longer term.

The postTAVI PPM implantation rate of $6 \%$ reported by this study was comparable to the rate of $2-51 \%$ reported in the literature. ${ }^{27}$ The finding that postTAVI PPM implantation was more common in advanced CKD patients than non-CKD patients also concurred with findings reported in the literature. ${ }^{16}$
It could partly be attributed to increased calcification that is commonly seen in CKD patients and is caused by hormonal and metabolic derangements such as increased parathyroid hormone, calcium-phosphate products and 1,25-dihydroxyvitamin D. ${ }^{28}$ During valve deployment, increased calcification in the left ventricular outflow tract could compress the conduction system and lead to conduction blockages that necessitate the need for PPM implantation.

In the literature, findings on the outcome of advanced CKD on valve haemodynamics are mixed. While some studies reported rapid deterioration in valve haemodynamics in advanced CKD patients, others ${ }^{29,30}$ did not report significant differences between these patients and non-CKD patients. ${ }^{13}$ Although this study did not find significant changes in AV area and mean gradient at 1 year, there were, however, 2 cases of 
"early" TAVI failure that had significant valve stenosis $<2$ years after TAVI was performed (Table 5). Both occurred in ESRF patients who were on dialysis and the phenomenon could be attributed to deranged and increased calcification. ${ }^{28}$ Larger long-term studies are needed to evaluate the clinical significance of early TAVI failure in dialysis patients.

PostTAVI, moderate AR was seen in more advanced CKD patients and was attributed to increased calcification. ${ }^{28}$ When calcification is present in the aortic annulus, it may prevent adequate sealing of the valve. Postoperative AR is not benign and has been identified as an independent predictor of all-cause and cardiovascular mortality after TAVI. ${ }^{31,32}$ It can affect the functional status of patients such as effort tolerance and trigger symptoms of heart failure. ${ }^{33}$

In this study, the findings of an AKI incidence of approximately $19 \%$ and CKD 4 patients with the highest risk of developing it were consistent with those reported in the literature. ${ }^{34,35}$ Findings of univariate analysis showed that AKI was a significant predictor of mortality, but its effect was attenuated by multivariate analysis. In their study, Allende et al reported that AKI was a significant predictor of overall mortality. ${ }^{17}$ A few reports had described renal trajectory and outcomes post-TAVI. In this study, the finding that CKD progression led to higher mortality at 3 months suggested that care should be taken to minimise AKI during: 1) the preoperative/perioperative phase through avoidance of haemodynamic instability and nephrotoxic agents but with provision of adequate hydration; and 2) the subacute and chronic phases post-TAVI to retard CKD progression since it heralds poorer long-term outcomes.

In this study, BEV had lower AV area and higher $\mathrm{AV}$ gradients with no differences in AR at 1 year compared to SEV, a result that was also reported by the CHOICE trial. ${ }^{36}$ However, the FRANCE-TAVI registry reported that SEV patients had higher risk of developing paravalvular leak than BEV patients and higher all-cause mortality at 2 years, irrespective of valve generation. ${ }^{37}$ These differences need to be validated in future studies.

A limitation of this study was the small sample size that limited extrapolation of its findings. The results will need to be validated in bigger patient cohorts. Since data was only available on renal trajectory at 3 months, more study is required to examine the long-term effects of renal disease progression. Nevertheless, this study had raised some interesting hypotheses and findings that can guide future research. Due to the non-randomised nature of the study, there is possibility of bias from confounding factors. The high incidence of valve degeneration in those who underwent TAVI was attributed to the predominant use of SAPIEN valves in the early phase of our TAVI programme.

\section{Conclusion}

In severe AS patients undergoing TAVI, CKD portends higher mortality and morbidity. In the long term, renal disease progression impacts negatively on outcomes. Dedicated preventive and management efforts should be undertaken to optimise outcomes in this group of patients.

\section{REFERENCES}

1. Leon MB, Smith CR, Mack M, Miller DC, Moses JW, Svensson LG, et al. Transcatheter aortic-valve implantation for aortic stenosis in patients who cannot undergo surgery. N Engl J Med 2010;363: 1597-607.

2. Smith CR, Leon MB, Mack MJ, Miller DC, Moses JW, Svensson LG, et al. Transcatheter versus surgical aortic-valve replacement in high-risk patients. N Engl J Med 2011;364:2187-98.

3. Leon MB, Smith CR, Mack MJ, Makkar RR, Svensson LG, Kodali SK, et al. Transcatheter or surgical aortic-valve replacement in intermediate-risk patients. N Engl J Med 2016;374:1609-20.

4. Low SKM, Sum CF, Yeoh LY, Tavintharan S, Ng XW, Lee SBM, et al. Prevalence of chronic kidney disease in adults with type 2 diabetes mellitus. Ann Acad Med Singapore 2015;44:164-71.

5. Rodés-Cabau J. Transcatheter aortic valve implantation: current and future approaches. Nat Rev Cardiol 2011;9:15-29.

6. Adams DH, Popma JJ, Reardon MJ, Yakubov SJ, Coselli JS, Deeb GM, et al. Transcatheter aortic-valve replacement with a self-expanding prosthesis. N Engl J Med 2014;370:1790-8.

7. Webb JG, Altwegg L, Masson JB, Al Bugami S, Al Ali A, Boone RA. A new transcatheter aortic valve and percutaneous valve delivery system. J Am Coll Cardiol 2009;53:1855-8.

8. Stevens PE, Levin A. Evaluation and management of chronic kidney disease: synopsis of the Kidney Disease: Improving Global Outcomes 2012 Clinical Practice Guideline. Ann Intern Med 2013;158:825-30

9. Mehta RL, Kellum JA, Shah SV, Molitoris BA, Ronco C, Warnock DG, et al. Acute Kidney Injury Network: report of an initiative to improve outcomes in acute kidney injury. Crit Care 2007; 11:R31.

10. Kappetein AP, Head SJ, Généreux P, Piazza N, van Mieghem NM, Blackstone $\mathrm{EH}$, et al. Updated standardized endpoint definitions for transcatheter aortic valve implantation: the Valve Academic Research Consortium-2 consensus document. Eur Heart J 2012;33:2403-18

11. Sinning JM, Ghanem A, Steinhäuser H, Adenauer V, Hammerstingl C, Nickenig G, et al. Renal function as predictor of mortality in patients after percutaneous transcatheter aortic valve implantation. JACC Cardiovasc Interv 2010;3:1141-9. 
12. Yamamoto M, Hayashida K, Mouillet G, Hovasse T, Chevalier B, Oguri A, et al. Prognostic value of chronic kidney disease after transcatheter aortic valve implantation. J Am Coll Cardiol 2013;62:869-77.

13. Dumonteil N, van der Boon RM, Tchetche D, Chieffo A, Van Mieghem NM, Marcheix B, et al. Impact of preoperative chronic kidney disease on short- and long-term outcomes after transcatheter aortic valve implantation: a Pooled-RotterdAm-Milano-Toulouse In Collaboration Plus (PRAGMATIC-Plus) initiative substudy. Am Heart J 2013;165:752-60.

14. Oguri A, Yamamoto M, Mouillet G, Gilard M, Laskar M, Eltchaninoff $\mathrm{H}$, et al. Impact of chronic kidney disease on the outcomes of transcatheter aortic valve implantation: results from the FRANCE 2 registry. EuroIntervention 2015;10:e1-9.

15. Ferro CJ, Chue CD, de Belder MA, Moat N, Wendler O, Trivedi $\mathrm{U}$, et al. Impact of renal function on survival after transcatheter aortic valve implantation (TAVI): an analysis of the UK TAVI registry. Heart 2015;101:546-52.

16. Gupta T, Goel K, Kolte D, Khera S, Villablanca PA, Aronow WS, et al. Association of chronic kidney disease with in-hospital outcomes of transcatheter aortic valve replacement. JACC Cardiovasc Interv 2017;10:2050-60.

17. Allende R, Webb JG, Munoz-Garcia AJ, de Jaegere P, Tamburino $\mathrm{C}$, Dager AE, et al. Advanced chronic kidney disease in patients undergoing transcatheter aortic valve implantation: insights on clinical outcomes and prognostic markers from a large cohort of patients. Eur Heart J 2014;35:2685-96.

18. Conrotto F, Salizzoni S, Andreis A, D’Ascenzo F, D’Onofrio A, Agrifoglio $M$, et al. Transcatheter aortic valve implantation in patients with advanced chronic kidney disease. Am J Cardiol 2017;119:1438-42.

19. Codner P, Levi A, Gargiulo G, Praz F, Hayashida K, Watanabe Y, et al. Impact of renal dysfunction on results of transcatheter aortic valve replacement outcomes in a large multicenter cohort. Am J Cardiol 2016;118:1888-96.

20. United States Renal Data System 2011 Annual Data Report. Morbidity and mortality in patients with chronic kidney disease. Am J Kidney Dis 2012;59:E59-68.

21. Dalrymple LS, Go AS. Epidemiology of acute infections among patients with chronic kidney disease. Clin J Am Soc Nephrol 2008;3:1487-93.

22. Kahn MR, Robbins MJ, Kim MC, Fuster V. Management of cardiovascular disease in patients with kidney disease. Nat Rev Cardiol 2013;10:261-73.

23. Go AS, Chertow GM, Fan D, McCulloch CE, Hsu CY. Chronic kidney disease and the risks of death, cardiovascular events, and hospitalization. N Engl J Med 2004; 351:1296-305.

24. Palmer SC, Di Micco L, Razavian M, Craig JC, Perkovic V, Pellegrini F, et al. Effects of antiplatelet therapy on mortality and cardiovascular and bleeding outcomes in persons with chronic kidney disease: a systematic review and meta-analysis. Ann Intern Med 2012;156:445-59.

25. Capodanno D, Angiolillo DJ. Antithrombotic therapy in patients with chronic kidney disease. Circulation 2012;125:2649-61.

26. D’Ascenzo F, Moretti C, Salizzoni S, Bollati M, D’Amico M, Ballocca F, et al. 30 days and midterm outcomes of patients undergoing percutaneous replacement of aortic valve according to their renal function: a multicentre study. Int J Cardiol 2013;167:1514-8.

27. Siontis GCM, Jüni P, Pilgrim T, Stortecky S, Büllesfeld L, Meier B, et al. Predictors of permanent pacemaker implantation in patients with severe aortic stenosis undergoing TAVR: a meta-analysis. J Am Coll Cardiol 2014;64:129-40.

28. Rattazzi M, Bertacco E, Del Vecchio A, Puato M, Faggin E, Pauletto P. Aortic valve calcification in chronic kidney disease. Nephrol Dial Transplant 2013;28:2968-76

29. Böning A, Boedeker RH, Rosendahl UP, Niemann B, Haberer S, Roth $\mathrm{P}$, et al. Long-term results of mechanical and biological heart valves in dialysis and non-dialysis patients. Thorac Cardiovasc Surg 2011;59:454-9.

30. Hahn HS, Maze SS. Rapid bioprosthetic valve degeneration in a patient undergoing hemodialysis. Ann Intern Med 2000;132:847.

31. Abdel-Wahab M, Zahn R, Horack M, Gerckens U, Schuler G, Sievert $\mathrm{H}$, et al. Aortic regurgitation after transcatheter aortic valve implantation: incidence and early outcome. Results from the German transcatheter aortic valve interventions registry. Heart 2011;97:899-906

32. Rajani R, Kakad M, Khawaja MZ, Lee L, James R, Saha M, et al. Paravalvular regurgitation one year after transcatheter aortic valve implantation. Catheter Cardiovasc Interv 2010;75:868-72.

33. Stähli BE, Maier W, Corti R, Lüscher TF, Jenni R, Tanner FC. Aortic regurgitation after transcatheter aortic valve implantation: mechanisms and implications. Cardiovasc Diagn Ther 2013;3:15-22.

34. Généreux P, Head SJ, Van Mieghem NM, Kodali S, Kirtane AJ, $\mathrm{Xu} \mathrm{K}$, et al. Clinical outcomes after transcatheter aortic valve replacement using Valve Academic Research Consortium definitions: a weighted meta-analysis of 3,519 patients from 16 studies. J Am Coll Cardiol 2012;59:2317-26.

35. Barbanti M, Latib A, Sgroi C, Fiorina C, De Carlo M, Bedogni F, et al. Acute kidney injury after transcatheter aortic valve implantation with self-expanding CoreValve prosthesis: results from a large multicentre Italian research project. EuroIntervention 2014;10133-40.

36. Abdel-Wahab M, Mehilli J, Frerker C, Neumann FJ, Kurz T, Tölg R, et al. Comparison of balloon-expandable vs self-expandable valves in patients undergoing transcatheter aortic valve replacement: the CHOICE randomized clinical trial. JAMA 2014;311:1503-14.

37. Van Belle E, Vincent F, Labreuche J, Auffret V, Debry N, Lefèvre T, et al. Balloon-expandable versus self-expanding transcatheter aortic valve replacement: a propensity-matched comparison from the FRANCE-TAVI registry. Circulation 2020;141:243-59. 